\title{
Assessment of Histo-Pathological Outcomes of Colorectal Cancer
}

\author{
Amir Khalil, Mohamad Nada, Ahmed Khalil, Essam Ebied \\ Department of General Surgery, Faculty of Medicine, Ain Shams University, Egypt
}

Background: Careful and accurate pathology reporting of colorectal cancer resection is vital because pathology reports are used to inform the prognosis, evaluate the quality of other clinical services, surgery and oncology and plan the treatment of patients. Objectives: To evaluate histopathological outcomes of resected colorectal specimens in terms of adequacy of resection, quality of pathological reports and the demographic data of the patients in El Demerdash Hospital in the period between June 2016 to June 2018.

Patients and methods: This is a retrospective study conducted at Ain Shams University Hospital by collecting the data from histopathological reports of 142 patients who underwent surgical resection and histopathological assessment of colorectal cancers.

Results: In our 2-year study period, 142 patients were included, mean age 52.95 years ( $56 \%$ male) and (43\% female). The most common site was ano-rectal tumors (30.3\%). (92.3\%) of pathology reports were fulfilling the criteria of qualified reports. Adequacy of resection in colon cancer was $(70.7 \%)$ and it was higher than that of rectal cancer (44\%). In the group of patients who received NAT there were $43.5 \%$ adequate LNS harvesting.

Conclusion: One of the most important prognostic factors in colorectal cancers is adequacy of resection. It was higher in colon cancer than that of rectal cancer. The commonest factor that affects the resection adequacy is the lymph node harvesting, which was apparently less in cases of rectal cancer. This may be attributed to the effect of neoadjuvant therapy on lymph nodes depletion, giving a false picture of inadequate resection.

Key words: Colorectal Cancer, Histopathological Assessment, Adequacy of Resection, Quality of Reports.

\begin{abstract}
Introduction
Colorectal Cancer (CRC) incidence and mortality rates vary markedly around the world. Globally, CRC is the third most commonly diagnosed cancer in males and the second in females, with 1.65 million new cases and almost 835,000 deaths in 2015. Rates are substantially higher in males than in females. ${ }^{1}$
\end{abstract}

Bleeding is the most common symptom of rectal cancer, occurring in $60 \%$ of patients, change in bowel habits is present in $43 \%$ of patients, Malaise is a nonspecific symptom and present in $9 \%$ of rectal cancer cases. Bowel obstruction due to a high-grade rectal lesion is rare, occurring in $9 \%$ of all cases. Pelvic pain is a late symptom, usually indicating nerve trunk involvement, and is present in $5 \%$ of all cases. Other manifestations include emergencies such as peritonitis from perforation $(3 \%)$ or jaundice, which may occur with liver metastases. $^{2}$

The definitive management for colorectal cancer is surgical either open or laparoscopic. Laparoscopicassisted colectomy was introduced 30 years ago. Perhaps $^{3}$ had not expected that laparoscopicassisted colectomy would be so widely used in the treatment of colon cancer. Just several years ago, a questionnaire survey in Great Britain and Ireland demonstrated that the number of surgeons using laparoscopic colorectal surgery for treatment of colorectal cancer more than doubled from 2004 to 2007. A total of $30 \%$ of these surgeons performed more than half of all their resections laparoscopically. ${ }^{4}$

Pathologic assessment of the colorectal cancer (CRC) resection specimens plays a central role in patient management. The pathology report informs prognosis and contributes to decisions regarding adjuvant therapy. Currently, the primary method for assessing prognostic differences among patients is the tumor-node-metastasis (TNM) staging system, developed by the American Joint Committee on Cancer (AJCC) and the International Union against Cancer (UICC). ${ }^{5}$

However there is significant variation in outcome of patients even within the same tumor stage. Many promising prognostic and/or predictive molecular and immune-histochemical biomarkers are emerging but morphological parameters are still important predictors of patient outcome. ${ }^{6}$

Many other histological features such as tumor budding, vascular invasion, peri-neural invasion, tumor grade and rectal tumor regression grade that may be of prognostic value are not part of TNM staging. Assessment of extramural tumor deposits 
and peritoneal involvement contributes to TNM staging but there are some difficulties with the definition of both of these features. ${ }^{7}$

Controversies in colorectal cancer pathology reporting include the subjective nature of some of the elements assessed, poor reporting rates and reproducibility and the need for standardized examination protocols and reporting. Molecular pathology is becoming increasingly important in prognostication and prediction of response to targeted therapies but accurate morphology still has a key role to play in colorectal cancer pathology reporting. 8

\section{Aim of the work}

To evaluate histopathological outcomes of resected colorectal specimens in terms of adequacy of resection, quality of pathological reports and the demographic data of the patients in El Demerdash Hospital in the period between June 2016 to June 2018.

\section{Patients and methods}

This is a retrospective study conducted at histopathology department of Ain Shams University hospital by collecting the data from histopathological reports of 142 patients who underwent surgical resection of colorectal cancers in colorectal unit in El-Demerdash hospital during the period from June 2016 to the end of June 2018.

\section{Inclusion criteria:}

All resected colorectal cancers in colorectal unit at El-Demerdash Hospital who underwent pathological assessment in El-Demerdash Hospital.

\section{Exclusion criteria:}

Pathology done outside El-Demerdash hospital.

Resected cancers outside Colo-rectal unit at ElDemerdash Hospital.

\section{Study procedures:}

- We collected the data of colorectal patients who underwent resection and pathological assessment in El-Demerdash Hospital in colorectal unit.

- Histopathological reports were revised in terms of adequacy of resection and quality of reports.

\section{Definition of adequacy of resections:}

- Proximal and distal margins: should be free.

- Circumferential margins: should be free.

- Lymph node retrieval: resected lymph nodes should be at least 12 lymph node.

\section{Items should be fulfilled in histopathological reports:}

- Full oncological data.

- Proximal and distal margins.
- $\quad$ Circumferential margins.

- $\quad$ Lymph nodes retrieval.

- Gross and microscopic pictures.

- Effect of neoadjuvant therapy.

- TNM classification.

- Lympho-vascular invasion.

Collected data were analyzed in excel sheet designed by me as a check list to facilitate data entry and retrieval.

\begin{tabular}{ll}
\hline I.D & Peri-neural .Invasion \\
Crystal N. & Longitudinal .Margins \\
Age & Circumferential margins \\
Sex & Deposits \& Invasion \\
Operation & Neo adj. treatment effect \\
Specimen & L.N.S \\
Length & Stagging \\
Tumor site & Adequacy of resections \\
Size & Quality of report \\
Histologic type and grade & Colon \\
L.V invasion & Rectum \\
\hline
\end{tabular}

Data were revised by statistics to determine the demographic data of patients, percentage of adequate resections and quality of reports.

\section{Results}

In our 2-year study period, 142 patients, with a mean age of 52.95 years, (56\% male) and (43\% female) with colorectal cancer met our selection criteria. The most common site is ano-rectal tumors (30.3\%), then left side (29.6\%) and finally right sided (28.2\%). Many procedure of colorectal resections were done according to the site of the lesion. Right hemicolectomy was the most common procedure that was done in 45 cases $(31.7 \%)$, then anterior resection $26(18.3 \%)$, abdominoperineal resection $20(14.1 \%)$, finally both left hemicolectomy and sigmoid colectomy accounts for (12.7\%). (Table 1).

Table 1: Site of tumor

\begin{tabular}{lcc}
\hline Tumor Site & No. & \% \\
\hline Right colon & 40 & $28.2 \%$ \\
Transverse colon & 15 & $10.6 \%$ \\
Left colon & 42 & $29.6 \%$ \\
Ano-rectal tumors & 43 & $30.3 \%$ \\
Part of colon & 2 & $1.4 \%$ \\
\hline
\end{tabular}

Different histological types were recorded in this study; the majority of cases were moderately differentiated adenocarcinoma (71.8\%) 102 out of 142 cases, followed by mucinous adenocarcinoma 33 cases $23.2 \%$. One of the prognostic criteria is the lympho-vascular invasion, this 
was detected in only 22 cases of this study, which represent about $15.5 \%$ of the total cases while Peri-neural invasion were recorded in 34 cases, while 107 cases were free, with a single case cannot be assessed. Other prognostic factors include tumor deposits. In this study, in $97(68 \%)$ cases no tumor deposits were detected while (29.6\%) were positive and missed data of other 3 cases.

In revision of adequacy of resection both longitudinal and circumferential margins should be free. In this study there were 132 (93\%) cases with free longitudinal margins while 10 cases were infiltrated. On the other hand 15 cases showed circumferential margin involvement with malignant cells which accounts for $(10.6 \%)$ of all the resected specimens. With regards to lymph node retrieval and adequacy of resection, resection of 12 or more lymph node is sign of adequate resection. In our study 100 (70.4\%) of resected specimen showed adequate lymph node resection.

Many Stages of the disease were recorded through our patients. $57 \%$ of the patients were in T3 stage and $14.1 \%$ were in T2 while $10.6 \%$ were in T4 stage. Precise and good histopathological assessment is very important in MDT and decision should be taken about the case. We found that $92.3 \%$ of our pathology reports were fulfilling the criteria of qualified reports but only $7.7 \%$ were missing some data (Table 2). One of the most important prognostic factors in colorectal cancers is adequacy of resection. In adequate resections both longitudinal and circumferential margins should be free and at least 12 lymph nodes should be resected. In our study $90(63 \%)$ patients had adequate resection and the remaining specimens showed in adequate resection. (Table $\mathbf{3}$ ).

Table 2: Quality of report

\begin{tabular}{llc}
\hline & & No. (\%) \\
\hline \multirow{2}{*}{ Quality of report } & Fulfilling & $131(92.3 \%)$ \\
& Missing & $11(7.7 \%)$ \\
\hline
\end{tabular}

Table 3: Adequacy of resection

\begin{tabular}{lcc}
\hline & No. (\%) \\
\hline \multirow{2}{*}{ Adequacy of resections } & Adequate & $90(63.4 \%)$ \\
& Inadequate & $52(36.6 \%)$ \\
\hline
\end{tabular}

On further analysis, we also found that incidence of colon cancer $(69.7 \%)$ was much higher than rectal cancer. Adequacy of resection in colon cancer was $(70.7 \%)$ and it was higher than that of rectal cancer adequacy of resection (44\%). (Table 4). In terms of adequacy of resection of colon in our study there was $(77.8 \%)$ adequate resection of lymph nodes (>12 LNS), (87.9\%) adequate resection to circumferential margins and (93.9\%) adequate resection to longitudinal margins. (Table 5).

On focusing on adequacy of resection in anorectal tumors we noticed according to lymph nodes harvesting that $53.5 \%$ show adequate resection. $83.7 \%$ showed adequate resection according to circumferential margin infiltration and $90.7 \%$ showed adequate resection according longitudinal margin infiltration Table. Adequacy of resection was less according to lymph nodes harvesting as (53.5\%) had pre-operative neo-adjuvant therapy which had big role on melting of lymph nodes and giving false low records of harvested lymph nodes. (Table 6).

\begin{tabular}{lc} 
Table 4: Incidence of colon and rectal \\
malignancy and adequacy of resection \\
\hline & No. (\%) \\
Colon & $99(69.7 \%)$ \\
Inadequate & $25(25.3 \%)$ \\
Adequate & $70(70.7 \%)$ \\
NA & $4(4.0 \%)$ \\
Rectum & $43(30.3 \%)$ \\
Inadequate & $23(53.5 \%)$ \\
Adequate & $19(44.2 \%)$ \\
NA & $1(2.3 \%)$ \\
\hline
\end{tabular}

The next table showed that there was higher incidence of infiltrated circumferential margins (20\%) and longitudinal margins (15\%) in patients who did not receive pre-operative treatment than those who received treatment (4.3\%) in both longitudinal and circumferential margins, but with no statistically significant difference between them may be due to lower number of patients. On the other hand, the number of free and adequate resection of lymph node decreased from $30 \%$ to $13 \%$ while the number of infiltrated and inadequate resection increased from $5 \%$ to $17.4 \%$ in patients who received neo-adjuvant therapy. This is all attributed to the effect of pre-operative treatment on lymph nodes shrinkage. (Table 7). 
Table 5: Adequacy of resection in colon cancer

\begin{tabular}{llc}
\hline & Colon & Total no. $=99$ \\
\hline & NA & $2(2.02 \%)$ \\
L.N infiltration & Free and adequate resection & $42(42.4 \%)$ \\
$\&$ harvesting & Free and inadequate resection & $14(14.1 \%)$ \\
& Infiltrated adequate resection & $35(35.4 \%)$ \\
& Infiltrated Inadequate resection & 6 \\
Circumferential Margins & NA & $(6.1 \%)$ \\
& Free & $(2.02 \%)$ \\
& Infiltrated & $87(87.9 \%)$ \\
\hline & Free & $(10.1 \%)$
\end{tabular}

Table 6: Anorectal tumors and adequacy of resection

\begin{tabular}{|c|c|c|c|}
\hline & & No. & $\%$ \\
\hline Ano-rectal tumors & & 43 & $100.0 \%$ \\
\hline \multirow{2}{*}{ Pre-operative Treatment } & No & 20 & $46.5 \%$ \\
\hline & Yes & 23 & $53.5 \%$ \\
\hline \multirow{5}{*}{ L.N infiltration } & Free and adequate resection & 9 & $20.9 \%$ \\
\hline & Free and inadequate resection & 14 & $32.6 \%$ \\
\hline & Infiltrated Adequate resection & 14 & $32.6 \%$ \\
\hline & Infiltrated Inadequate resection & 5 & $11.6 \%$ \\
\hline & Not applicable & 1 & $2.3 \%$ \\
\hline \multirow[t]{2}{*}{ Circumferential Margins } & Free & 36 & $83.7 \%$ \\
\hline & Infiltrated & 7 & $16.3 \%$ \\
\hline \multirow{2}{*}{ Longitudinal Margins } & Free & 39 & $90.7 \%$ \\
\hline & Infiltrated & 4 & $9.3 \%$ \\
\hline
\end{tabular}


Table 7: Effect of pre-operative treatment on margins and lymph nodes infiltration

\begin{tabular}{|c|c|c|c|c|c|c|c|c|}
\hline \multirow{3}{*}{ Ano-rectal tumors } & \multicolumn{5}{|c|}{ Pre-operative treatment } & \multirow{3}{*}{$\begin{array}{l}\text { Test } \\
\text { value }\end{array}$} & \multirow{3}{*}{ P-value } & \multirow{3}{*}{ Sig } \\
\hline & \multirow{2}{*}{$\begin{array}{l}\text { No (20) pt. } \\
\text { No. }\end{array}$} & \multicolumn{4}{|c|}{ Yes (23) pt. } & & & \\
\hline & & $\%$ & No. & $\%$ & & & & \\
\hline \multirow{4}{*}{ L.N infiltration $\&$ adequate } & Free and adequate resection & 6 & $30.0 \%$ & 3 & $13.0 \%$ & 1.859 & 0.173 & NS \\
\hline & Free and inadequate resection & 5 & $25.0 \%$ & 9 & $39.1 \%$ & 0.973 & 0.324 & NS \\
\hline & Infiltrated Adequate resection & 7 & $35.0 \%$ & 7 & $30.4 \%$ & 0.102 & 0.749 & NS \\
\hline & Infiltrated Inadequate resection & 1 & $5.0 \%$ & 4 & $17.4 \%$ & 1.598 & 0.206 & NS \\
\hline \multirow[t]{3}{*}{ resection } & Not applicable & 1 & $5.0 \%$ & 0 & $0.0 \%$ & 1.177 & 0.278 & NS \\
\hline & Adequate resection & 13 & $65.0 \%$ & 10 & $43.5 \%$ & 1.992 & 0.158 & NS \\
\hline & Inadequate resection & 6 & $30.0 \%$ & 13 & $56.5 \%$ & 3.051 & 0.081 & NS \\
\hline \multirow{2}{*}{ Circumferential Margins } & Free & 16 & $80.0 \%$ & 22 & $95.7 \%$ & \multirow{2}{*}{2.550} & \multirow{2}{*}{0.110} & \multirow{2}{*}{ NS } \\
\hline & Infiltrated & 4 & $20.0 \%$ & 1 & $4.3 \%$ & & & \\
\hline Longitudinal Margins & Free & 17 & $85.0 \%$ & 22 & $95.7 \%$ & 1.439 & 0.230 & NS \\
\hline
\end{tabular}

This table showed that the number of infiltrated lymph nodes in patients who received neo-adjuvant therapy fell from $35.0 \%$ to $30.4 \%$ but lymph node inadequacy of resection increased in patients who received pre-operative treatment from $30 \%$ to $56.5 \%$. (Table 7).

Finally, In anorectal cancer, the group of patients who didn't receive NAT there were adequate resection to lymph nodes (LNS) in $65.0 \%$, free longitudinal margins in $85 \%$ and free circumferential margins in $80 \%$ of cases. In the other group of patients who received NAT there were $43.5 \%$ adequate LNS harvesting and $95.7 \%$ free both longitudinal and circumferential margins. (Table 7).

\section{Discussion}

Precise information and histopathological assessment about resected specimens of large gut cancer was necessary for prognosis and management. The previous investigation showed that this was not consistently achieved in the relatively simple assessment of this common tumor. ${ }^{9}$

This study included 142 patients who underwent any type of colorectal resection for their malignant lesions. Patient's ages ranged from 17 up to 80 years old with the mean age 52.9 year. This mean of age was less than that observed in other studies as in Hochster et al. ${ }^{10}$ who studied about 21,925 patients with mean age 63 year, also less than Zafar et al. ${ }^{11}$ with 64 years old mean age of 682 patients and close to Ghahramani et al. ${ }^{12}$ who studied 250 patients with mean age 54 years old. That may indicate that the incidence of colorectal cancers occurs in younger age group in Egypt.

Gender distribution in this study showed males prevalence (80 out of 142 ) $56.3 \%$, which was comparable to the finding of Murphy et al. ${ }^{13}$ who studied 927 cases with $54.2 \%$ male predominance and Johnson et al. ${ }^{14}$ who studied 569 casees with also $54 \%$ but slightly less than Brule et al. ${ }^{15} 60.9 \%$ male predominance. This was also higher that Tonini et al. ${ }^{16}$ who revealed slight male predominance by $50.8 \%$ which may suggest the incidence of cancer colon was slightly higher in males, we didn't find any studies showing female predominance in cancer colon.

According to tumor location, the most common site in this study is ano-rectal tumors which accounted for $30.3 \%$, This figure was exactly like Gravante et al. ${ }^{17}$ who reported (30.4\%) of rectal tumors of total colo-rectal cancer cases. The left colon, including tumor located from the splenic flexure down just before the recto-sigmoid junction, represented about $29.6 \%$ of the total cases. This figure was approximate to the prevalence of left colonic tumors location represented in other studies of large group of patients as in Siegel et al. ${ }^{18}$ who worked on more than 132000 patients but less than Johnson et al. ${ }^{14}$ who published higher incidence rate $60 \%$ of left side large gut cancers.

Regarding surgical treatment we could observe that the right hemicolectomy was the most common operation $31.7 \%$ in this study. This was consistent with Tonini et al. ${ }^{16}$ who reported the highest incidence for right hemicolectomy 39\% and Gravante et al. ${ }^{17}$ with $39.7 \%$. Although the left 
colon was the higher incidence site, this could be explained by the fact that the left colon cancer has a variety of procedures, include left hemicolectomy, sigmoidectomy or even anterior resection. Also the right hemicolectomy was also performed in some cases of transverse colon cancer.

Histopathological examination revealed that 102 cases (about $72 \%$ ) were moderately differentiated adenocarcinoma compared to $(74.9 \%)$ reported by Shen et al., ${ }^{19}$ while mucinous type of adenocarcinoma was reported in 33 cases (23\%) as the 2 nd most common pathological type, This was consistent with the study of Catalano et al. ${ }^{20}$ who examined 255 patient's specimens, $19 \%$ of them was mucinous adenocarcinoma.

One of the important prognostic criteria is the lympho-vascular invasion, this was detected in only 22 cases of this study, which represent about $15.5 \%$ of the total cases. This percentage was less than Betge et al..$^{21}$ who reported $23 \%$ of vascular invasion and $33 \%$ of lymphatic invasion and less than Higgins et al. ${ }^{22}$ who detected vascular invasion in $62(48.1 \%)$ of specimens. In another study Courtney et al. ${ }^{23}$ reported that $28 \%$ of their cases were positive for lympho-vascular invasion. This may indicate early detection of the disease in our study.

Many Stages of the disease were recorded through our series. $57 \%$ of the patients were in T3 stage and $14.1 \%$ were in T2 while $10.6 \%$ were in T4 stage. These figures were in agreement with Tonini et al. ${ }^{16}$ who reported an incidence of T3 of (53.3\%) among the resected colorectal cancers out of 643 specimen. It was also close to Amajoyi et al. ${ }^{24}$ who noticed $50 \%$ of 502 patients were in T3 stage, $23 \%$ in $\mathrm{T} 2$ and $6 \% \mathrm{~T} 4$ tumors.

With regards to resection margins infiltration, our study showed that there were 132 (93\%) cases with free longitudinal margins while 10 cases were infiltrated. On the other hand 15 cases show circumferential margin involvement with malignant cells which accounts for (10.6\%) of all the resected specimens and $87.3 \%$ had adequate resection. Approximately, that value was equal to Tonini et al. ${ }^{16}$ who reported $87.3 \%$ of 615 patients with free resection margins.

The reports were analyzed for completeness according to 10 key prognostic features included in the College of American Pathologists (CAP) checklist for the reporting of colorectal cancer. These features included tumor size (in greatest dimension), TNM stage, histologic type, histologic grade, circumferential radial margin (CRM) involvement, distance to the CRM, lympho-vascular invasion (LVI), extramural venous invasion (EMVI), PNI, and regional tumor deposits ${ }^{25}$ as well as some points added by our team.

A good quality histopathology report will facilitate decision for postoperative adjuvant therapy and reliably predicts outcome in patients with colorectal cancer. We noticed that $92.3 \%$ of our pathology reports were fulfilling the criteria of qualified reports giving the full data needed. This is higher than the results shown by Rigby et al. ${ }^{26}$ who founded that $18 \%$ had one or more items missing from their reports.

According to National Comprehensive Cancer Network (NCCN) guidelines and American Joint Committee on Cancer (AJCC) about adequacy of resection in CRC, it is considered adequately resected specimens that showed free longitudinal and circumferential surgical margins in addition to excision of at least 12 lymph nodes Tonini et al. ${ }^{16}$ Adequate resection was obtained in about $63 \%$ of all cases included in this study, this figure was close to the results published by Cianchi et al.,27 which showed that $60.3 \%$ of 551 cases were adequately resected. Other studies showed a better percentage of adequate resection, as in Senthil et al. ${ }^{28}$ who achieved $74 \%$ and Gravante et al. ${ }^{17}$ who presented $69.1 \%$.

The extent of lymph node involvement is the most important prognostic factor in resected locoregional colorectal cancer. $42.2 \%$ of our total study cases showed lymph nodes infiltration. This rate of infiltration was higher than Johnson et al. ${ }^{14}$ who recorded $(38 \%)$ of 219 patients had one or more positive lymph nodes identified. This result is also higher than Tonini et al. ${ }^{16}$ who had $40 \%$ of positive lymph nodes infiltration.

Currently, examination of at least 12 lymph nodes is recommended for adequate colo-rectal cancer staging. We noticed that $70.4 \%$ of total cases showed adequate LNS resection and that was slightly more than Ghahramani et al. ${ }^{12}$ who recorded $69 \%$ of adequate resection of lymph nodes in total number of cases of 250 patients. Also it was marginally less than Onitilo et al. ${ }^{29}$ who recorded $74 \%$ of adequate lymph node resection. This was however much higher than Johnson et al. ${ }^{14}$ that recorded that (22\%) of 569 underwent an adequate harvest of lymph nodes.

In terms of adequacy of resection of colon in our study, there was adequate resection in colon cancer $(70.7 \%)$ also $(77.8 \%)$ adequate resection of lymph nodes ( $>12$ LNS) $(87.9 \%$ ) adequate resection to circumferential margins and (93.9\%) adequate resection to longitudinal margins. Adequate resection of lymph nodes in our case series was less than Higgins et al. ${ }^{22}$ who noted only $17(10.2 \%)$ of 
specimens containing $<12$ nodes of 167 patients.

Specifically in rectal resections, anterior resection or APR, the percentage of adequate resection decreased to be about $44 \%$ of cases, regardless of the reason of inadequacy, less than the colon adequacy of resection of colonic specimens of $70.7 \%$. These figures are lower in comparison to $45.4 \%$ and $59.1 \%$ of cases respectively noted by Gravante et al. ${ }^{17}$ The commonest factor contributing to the percentage of inadequate resection is lymph node harvesting.

With regards to anorectal tumors, our study showed that, according to lymph nodes retrieval $53.5 \%$ show adequate resection compared to $72 \%$ recorded by Amajoyi et al. ${ }^{24} 83.7 \%$ showed adequate resection according to circumferential margin infiltration and $90.7 \%$ showed adequate resection according longitudinal margin infiltration.

The same study also revealed that there was higher incidence of infiltrated circumferential margins (20\%) and longitudinal margins (15\%) in patients who did not receive neo-adjuvant therapy compared with those who received treatment $(4.3 \%)$ in both longitudinal and circumferential margins, this difference however was not statistically significant but this may be due to lower number of patients in their series. The study also demonstrated that there were adequate resection in lymph nodes (more than 12 LNS) in rectal cancer in patients who didn't receive NAT in $(65 \%)$ which was less than Amajoyi et al. ${ }^{24}$ who previously stated that the percentage of patients with at least 12 nodes in the non-NAT group was $72 \%$. This rate of adequate LNS resection dropped to be $43 \%$ in our study in the group of patients who underwent NAT.

On the other hand, the number of infiltrated and adequate resection of lymph node decreased from $35 \%$ to $30.4 \%$ while the number of free and inadequate resection increased from $25 \%$ to $39.1 \%$ in patients who received neo-adjuvant therapy. This was all attributed to the effect of pre-operative treatment on lymph nodes depletion.

In another way, patients who received neo-adjuvant treatment showed a higher incidence of inadequate lymph node resection from $30 \%$ in the no treatment arm to $56.5 \%$ in the treatment group. This is possibly due to its effect on nodal lymphocyte depletion with atrophy and fibrosis of the stroma, shrinkage and melting of the surrounding lymph nodes, which cannot be counted during histopathological assessment, giving a false picture of inadequate resection. These findings likely reflected significant down staging in the preoperative RT patients.

This was also confirmed by Baxter et al. ${ }^{30}$ who founded that lymph node staging (LNS) that met the conventional criterion for adequacy (i.e., at least 12 nodes) was less likely after preoperative RT. Using that criterion, $33 \%$ of patients with rectal cancer who did not undergo preoperative RT had undergone adequate LNS compared with $20 \%$ who had undergone preoperative RT.

Finally, in terms of adequacy of lymph nodes resection in rectal cancer, the recommendations for adequate L.N.S resection in patients who underwent NAT should be re-evaluated in a different way than those patients who did not received NAT.

\section{Conclusion}

Precise information about resected specimens of large gut cancer is necessary for prognosis and management. One of the most important prognostic factors in colorectal cancers is adequacy of resection. It was higher in colon cancer than that of rectal cancer. The commonest factor that affects the resection adequacy is the lymph node harvesting, which was apparently less in cases of rectal cancer. This may be attributed to the effect of neoadjuvant therapy on lymph nodes shrinkage and depletion, which cannot be counted during histopathological assessment, giving a false picture of inadequate resection. These findings likely reflected significant down staging in the preoperative RT patients. Finally, this indicates considering new recommendations in assessment of adequate LNS retrieval in rectal patients who receive NAT.

\section{Conflict of interest}

Dr. Khalil has nothing to disclose.

\section{References}

1. Fitzmaurice C, Allen C, et al: Global, Regional, and National Cancer Incidence, Mortality, Years of Life Lost, Years Lived With Disability, and Disability-Adjusted Life-years for 32 Cancer Groups, 1990 to 2015: A systematic analysis for the global burden of disease study. JAMA Oncol. 2017; 3: 524.

2. Kemppainen M, Raiha I, Rajala $T$, et al: Characteristics of colon cancer in elderly patients. Gerontology. 1993; 39: 222-227.

3. Jacobs M, Verdeja JC, Goldstein HS: Minimally invasive colon resection (laparoscopic colectomy). Surg Laparosc Endosc. 1991; $1: 144-150$.

4. Schwab KE, Dowson HM, Van Dellen J, et al: The uptake of laparoscopic colorectal surgery in Great Britain and Ireland: A questionnaire survey of consultant members of the ACPGBI. Colorectal Dis 2009; 11: 318-322. 
5. Shepherd NA, Baxter KJ, Love SB: The prognostic importance of peritoneal involvement in colonic cancer: A prospective evaluation. Gastroenterology. 2007; 112: 1096-1102.

6. Lennon AM, Mulcahy $H E$, Hyland $J M$, et al: Peritoneal involvement in stage II colon cancer. Am J Clin Pathol. 2013; 119: 108-113.

7. Sobin LH, Gospodarowic MK, Wittekind C, editors. International Union Against Cancer (UICC). TNM classification of malignant tumours. $7^{\text {th }}$ ed. New York: Wiley-Blackwell. 2009.

8. Puppa G, Sonzogni A, Colombari R, Pelosi G: TNM staging system of colorectal carcinoma: a critical appraisal of challenging issues. Arch Pathol Lab Med. 2010; 134: 837-852.

9. Loughrey MB, Quirke P, Shepherd NA: Dataset for histopathological reporting of colorectal cancer. Document number G049 in: Standards and datasets for reporting cancers. 2017)." The Royal College of Pathologists website (http://www. rcpath. org.) https://www. rcpath. org/profession/ publications/cancer-datasets. html. Accessed January 6 (2018).

10. Hochster HS, Lau A, Turner $M$, et al: Age distribution of tumor gene expression in patients with stage II/III Colon Cancer. 2018; 552-552.

11. Zafar SY, Abernethy AP, Abbott DH, et al: Comorbidity, age, race and stage at diagnosis in colorectal cancer: A retrospective, parallel analysis of two health systems. BMC Cancer. 2008; 8(1): 345.

12. Ghahramani L, Razzaghi S, Mohammadianpanah $M$, et al: Adequacy of lymph node staging in colorectal cancer: analysis of 250 patients and analytical literature review. Annals of Colorectal Research. 2013; 1(1): 3-1.

13. Murphy G, Devesa SS, Cross AJ, et al: Sex disparities in colorectal cancer incidence by anatomic subsite, race and age. International Journal of Cancer. 2011; 128(7): 1668-75.

14. Johnson PM, Malatjalian D, Porter GA: Adequacy of nodal harvest in colorectal cancer: A consecutive cohort study. Journal of Gastrointestinal Surgery. 2002; 6(6): 883-90.

15. Brule SY, Jonker DJ, Karapetis CS, et al: Location of colon cancer (right-sided versus left-sided) as a prognostic factor and a predictor of benefit from cetuximab in NCIC CO. 17. European Journal of Cancer. 2015; 51(11): 1405-14.

16. Tonini V, Birindelli A, Bianchini S, et al: Factors affecting the number of lymph nodes retrieved after colo-rectal cancer surgery: A prospective single-centre study. The Surgeon. 2020; 18(1): 31-6.

17. Gravante G, Parker R, Elshaer M, et al: Lymph node retrieval for colorectal cancer: Estimation of the minimum resection length to achieve at least 12 lymph nodes for the pathological analysis. International Journal of Surgery. 2016; 25: 153-7.

18. Siegel RL, Ward EM, Jemal A: Trends in colorectal cancer incidence rates in the United States by tumor location and stage, 1992-2008. Cancer Epidemiology and Prevention Biomarkers. 2012; 21(3): 411-6.

19. Shen SS, Haupt BX, Ro JY, et al: Number of lymph nodes examined and associated clinicopathologic factors in colorectal carcinoma. Archives of pathology \& laboratory medicine. 2009; 133(5): 781-6.

20. Catalano V, LoupakisF, Graziano F, etal: Mucinous histology predicts for poor response rate and overall survival of patients with colorectal cancer and treated with first-line oxaliplatinand/or irinotecan-based chemotherapy. British journal of cancer. 2009; 100(6): 881-7.

21. Betge J, Pollheimer $M J$, Lindtner RA, et al: Intramural and extramural vascular invasion in colorectal cancer: prognostic significance and quality of pathology reporting. Cancer. 2012; 118(3): 628-38.

22. Higgins $P$, Nemeth $T$, Bennani $F$, et al: The adequacy of lymph node clearance in colon cancer surgery performed in a non-specialist centre; implications for practice. Irish Journal of Medical Science (1971-). 2020; 189(1): 75-81.

23. Courtney ED, West NJ, Kaur C, et al: Extramural vascular invasion is an adverse prognostic indicator of survival in patients with colorectal cancer. Colorectal Disease. 2009; 11(2): 150-6.

24. Amajoyi R, Lee $Y$, Recio PJ, Kondylis PD: Neoadjuvant therapy for rectal cancer decreases the number of lymph nodes harvested in operative specimens. The American Journal of Surgery. 2013; 205(3): 289-92.

25. Messenger DE, McLeod RS, Kirsch R: What impact has the introduction of a synoptic report for rectal cancer had on reporting outcomes for specialist gastrointestinal and nongastrointestinal pathologists?. Archives of Pathology \& Laboratory Medicine. 2011; 135(11): 1471-5.

26. Rigby K, Brown SR, Lakin G, et al: The use of a 
proforma improves colorectal cancer pathology reporting. Annals of the Royal College of Surgeons of England. 1999; 81(6): 401.

27. Cianchi F, Cortesini C, Trallori G, et al: Adequacy of lymphadenectomy in laparoscopic colorectal cancer surgery: A single-centre, retrospective study. Surgical Laparoscopy Endoscopy \& Percutaneous Techniques. 2012; 22(1): 33-7.

28. Senthil M, Trisal V, Paz IB, et al: Prediction of the adequacy of lymph node retrieval in colon cancer by hospital type. Archives of Surgery.
2010; 145(9): 840-3.

29. Onitilo AA, Stankowski RV, Engel JM, et al: Adequate lymph node recovery improves survival in colorectal cancer patients. Journal of Surgical Oncology. 2013; 107(8): 828-34.

30. Baxter NN, Morris AM, Rothenberger DA, et al: Impact of preoperative radiation for rectal cancer on subsequent lymph node evaluation: a population-based analysis. International Journal of Radiation Oncology* Biology* Physics. 2005; 61(2): 426-31. 